\section{Late Distal Perigraft Endoleak after Endovascular Repair of an Abdominal Aortic Aneurysm due to Cranial Migration of the Iliac Branch of a Modular Stent-Graft}

Reperfusion of the aneurysmal sac after endovascular implantation of an aortic stentgraft (also called "endoleak") is probably the major failure mode of this new technique. An endoleak may be caused by a variety of factors and can originate perioperatively or can develop later on. In the presented case the endoleak developed 4 years after stentgraft implantation due to a cranial migration and folding of the iliac limb into the aneurysmal sac. To the best of our knowledge, this particular late stentgraft migration, which led to a major endoleak, is not yet described in literature.

\section{Case Report}

A 64-year-old man underwent 15 years ago an aortobiiliac endarterectomy for the treatment of bilateral claudication due to aortoiliac occlusive disease. 4 years ago, an AAA was incidentally found by ultrasound and confirmed by spiral computed tomography (CT). The abdominal spiral CT scan showed an infrarenal aortic aneurysm originating $7.5 \mathrm{~cm}$ below the origin of the ostium of the lowermost renal artery and extending to the aortic bifurcation. The aneurysmal sac presented a circumferential thrombus and had a maximal diameter of $50 \mathrm{~mm}$ (Fig. 1). The measured dimensions of the AAA and its favorable relationship to the visceral and iliac arteries made an endovascular repair possible by implantation of a modular stent-graft (Stentor, MinTec, La Ciotat, France). The body of the stent-graft was introduced after left femoral cutdown through an $18 \mathrm{~F}$ sheath and the contralateral right iliac limb was correctly implanted by percutaneous way through a $10 \mathrm{~F}$ sheath. Because of additional stenoses at both external iliac arteries, self-expandable bare stents (Wallstent, Schneider, Bülach, Switzerland) were also inserted to obtain a correct iliac run-off. Perioperative control angiography showed correct positioning and patency of the modular stent-graft without any endoleak. Follow-up was performed either by physical examination and by spiral CT scan, after 1 week, 3, 6, 12, 24 and 36 months. The consecutive spiral CT-scans showed a complete exclusion of the aneurysm and good patency of the stent-graft. We also noted a gradual decrease of the maximal diameter of the thrombosed aneurysmal sac, which was $50 \mathrm{~mm}$ immediately after exclusion, to $30 \mathrm{~mm}$ after 3 years (Fig. 2). Control physical examination 4 years after stent-graft implantation revealed a pulsatile mass and spiral CT scan

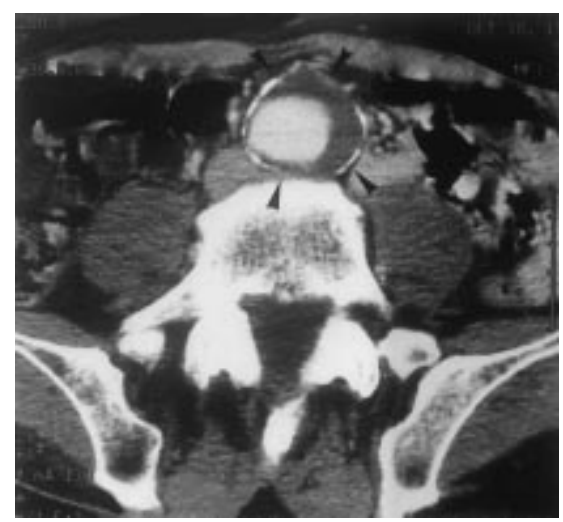

Fig. 1 Preoperative CT scan shows the AAA (arrowheads) with a maximal diameter of $50 \mathrm{~mm}$. Note the circumferential mural thrombus.

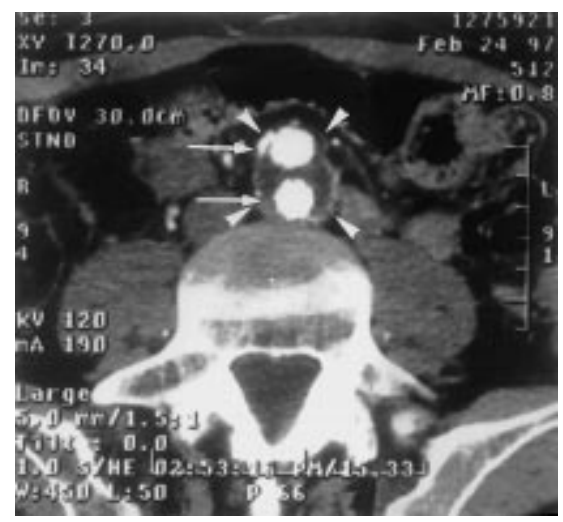

Fig. 2 Control CT scan 2 years after endovascular repair of the AAA illustrates the progressive decrease in maximal diameter of the excluded aneurysm (arrowheads) and the absence of endoleak into the thrombosed aneurysmal sac. Note the good opacification of both iliac limbs of the stent-graft (arrows). confirmed the reperfusion of the aneurysmal sac and the increase of the aneurysmal volume to a maximal diameter of $45 \mathrm{~mm}$ (Fig. 3). Abdominal plain film followed by aortography (Fig. 4) clearly showed a cranial migration of the distal end of the right iliac limb, which is also kinked and folded into the reperfused aneurysmal sac. There was a correct position and patency of the aortoiliac stent-graft component. Because of the migration and folding of

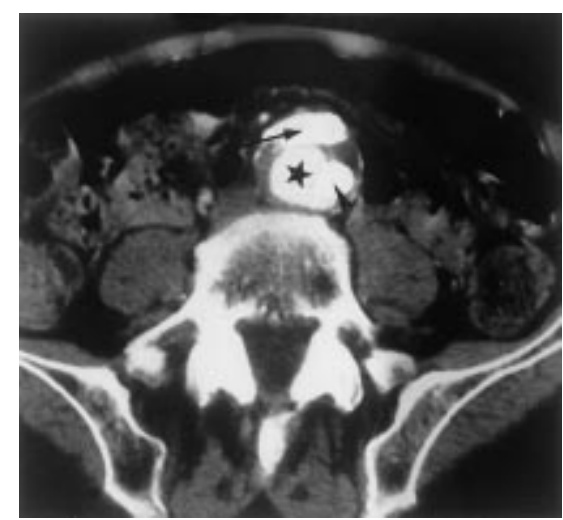

Fig. 3 Control CT scan 4 years after endovascular repair of the AAA clearly shows the right (arrow) and the left (arrowhead) iliac limb of the stent-graft, but also the major endoleak (asterisk) located in between the right and the left limb. Note also the increase in diameter of the centrally reperfused aneurysmal sac.

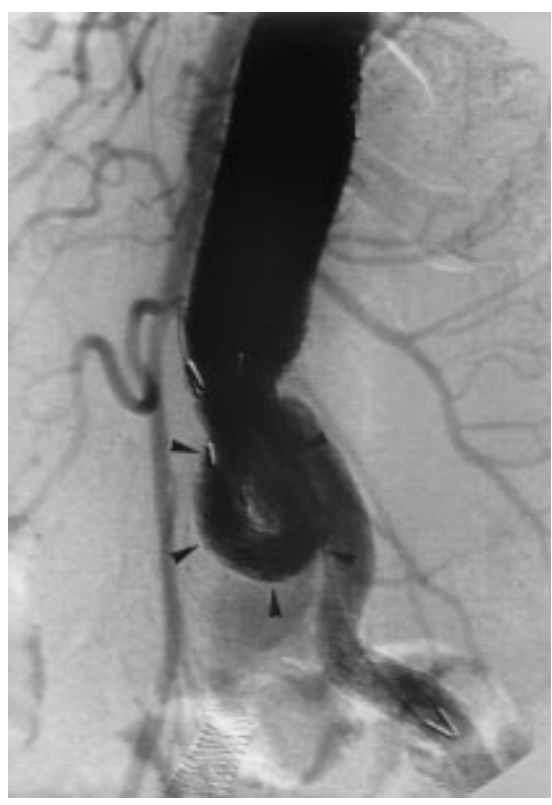

Fig. 4 An angiography reveals the cranial migration and folding of the right iliac stentgraft (arrowheads) into the reperfused aneurysmal sac. 
the iliac stentgraft into the reperfused aneurysmal sac, definitive treatment of this late complication could only be done by open surgical repair.

\section{Discussion}

Successful endovascular treatment of an AAA depends on its complete and durable exclusion from the arterial circulation. When the exclusion of the AAA is incomplete, an endoleak develops and, when untreated, may cause aneurysmal rupture (Torsello et al., J Vasc Surg 1998; 28: 184). Endoleaks have been reported to complicate between $8 \%$ and $44 \%$ of all AAA repairs. Depending on the origin of the endoleak, White et al. (White et al., J Endovasc Surg 1997; 4: 152) described a clear classification containing 4 subgroups. Type I is present when a persistent perigraft channel of blood flow develops due to inadequate or ineffective seal at the proximal or distal graft ends. Type II endoleak occurs when there is persistent collateral blood flow into the aneurysmal sac flowing retrogradely from patent lumbar arteries, inferior mesenteric arteries (Van Schie et al., J Endovasc Surg 1997; 4: 312) or other collateral vessels. Type III endoleak arises at the midgraft region due to leakage through a defect in the graft fabric or between segments of a modular stent-graft (Maleux et al., J Vasc Surg 1998; 28: 349). Type IV endoleak is seen on completion angiography or subsequent contrast studies as any minor blush of contrast that is presumed to emanate from blood diffusion across the pores of a highly porous graft fabric. Endoleak of undefined origin is diagnosed when the precise source of endoleak will not be clear from routine follow-up imaging studies.

When an endoleak is present since the perioperative period, it is called "primary endoleak", when there is a late reperfusion of the aneurysm after initial successful exclusion, it is called "secondary endoleak". In our case a late (secondary) type I (distal perigraft) endoleak is presented. The cause of the late reperfusion was not distal progression of the aneurysm, but a cranial migration of the distal part of the right iliac limb. The migration is pronounced by the fact that the distal part of the iliac branch is flipped over $180^{\circ}$ cranially, so that this iliac branch became completely and horizontally positioned in the reperfused aneurysmal sac. The reason why the migration oc- curred 4 years after successful stentgraft implantation is still unclear, but in agreement with White et al. (White et al., J Vasc Surg 1997; 26: 133), we presume that regression of the thrombosed aneurysm can cause important forces on the stent-graft, provoking cranial or distal migration of (a part of) the stentgraft, a phenomenon which might also provoke a late type III endoleak (Maleux et al., J Vasc Surg 1998; 28: 349).

The presence of barbs or other fixation material at the distal ends of the stentgraft should prevent this late complication. Treatment of this late complication is mandatory and most likely consists of conversion to open repair.
In summary, we present a late distal perigraft (type I) endoleak due to cranial migration of the contralateral iliac branch of a modular stent-graft. This late type I endoleak cannot be treated by any percutaneous technique because of the fact that the iliac limb not only migrated cranially, but also flipped entirely into the reperfused aneurysmal sac and finally took a nearly horizontal position in the sac.

G. Maleux, A. Nevelsteen, L. Stockx, Leuven, Belgium 